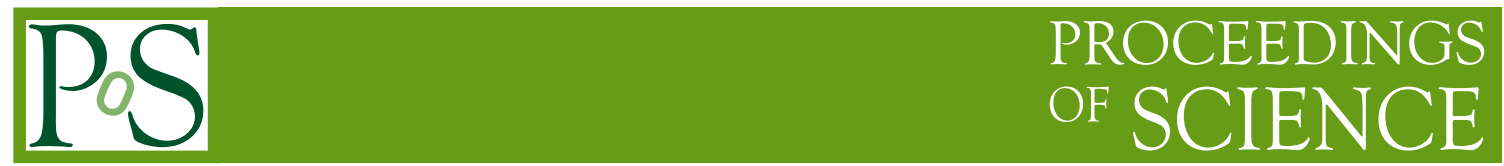

\title{
Measurement of Cosmic Deuteron Flux with the AMS-02 Detector
}

F Dimiccoli, ${ }^{a, *}$ R Battiston, ${ }^{a}$ I Lazzizzera, ${ }^{a}$ F Nozzoli ${ }^{a}$ and P Zuccon ${ }^{a, b}$

${ }^{a}$ INFN, Trento Institute for Fundamental Physics and Applications, I-38123, Trento, Italy

${ }^{b}$ Physics, Università degli Studi di Trento, I-38123 Trento, Italy

E-mail: francesco.dimiccoli@unitn.it

The deuteron flux in cosmic rays is one of the most valuable tools for understanding the propagation of $\mathrm{CR}$ in the galaxy and constrain the models that describe it. In this work, a preliminary measurement of D flux is presented, obtained from the data of the AMS-02 experiment, considering a data-taking period of 7 years (2011-2018).

40th International Conference on High Energy physics - ICHEP2020

July 28 - August 6, 2020

Prague, Czech Republic (virtual meeting)

\footnotetext{
${ }^{*}$ Speaker
} 


\section{Introduction}

The measurement of light isotopic components in cosmic rays (CR) like deuterons (D) is extremely important because of their production mechanism. They are spallation reactions of primary $\mathrm{CR}$ protons and ${ }^{4} \mathrm{He} \mathrm{nu}-$ clei on the protons of the Inter Stellar Medium (ISM). For this reason, the measurement of these components and of the ratio to their respective primary can provide important constrains about the $\mathrm{CR}$ residence time within galaxy and their propagation history. [1,2].

In general, light isotopes originate from the spallation of heavier ones, for example D from ${ }^{3} \mathrm{He}$ and ${ }^{4} \mathrm{He}$. The deuteron case is special though, since it can be produced also by p-p fusion at extremely low energies (below $1 \mathrm{GeV} / \mathrm{n})$. Their abundance, provide additional and complementary information with respect to the most common tool used for propagation studies, the Boron over Carbon $(\mathrm{B} / \mathrm{C})$ flux ratio. Deuteron, being a $\mathrm{Z}=1$ nucleus, spans relatively larger propagation distances with respect to Boron and Carbon, therefore it is a complementary probe to test the propagation parameters necessary to de-

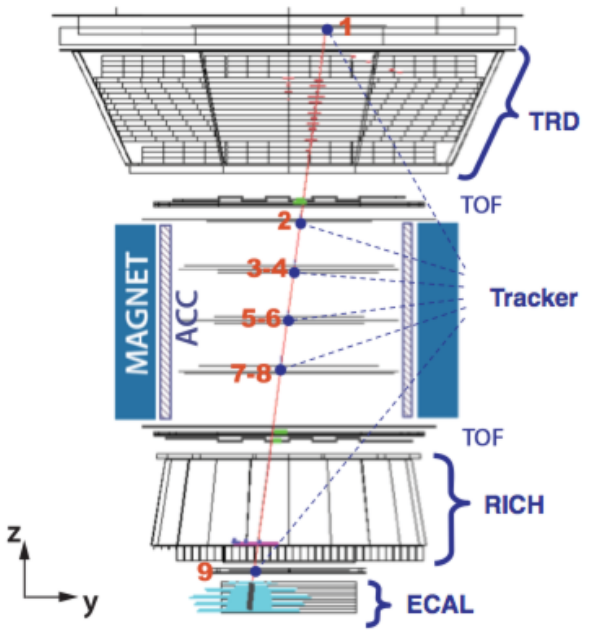

Figure 1: Scheme of the AMS-02 detector. From the top, tracker first layer (1), TRD, upper ToF, inner tracker layers $(2,3,4,5,6,7,8)$, lower ToF, RICH, tracker layer 9 and ECAL. Sixteen curved scintillator panels (Anti-Coincidence Counters, ACC) surround the inner tracker inside the $0.14 \mathrm{~T}$ magnet bore. termine the flux of secondary produced anti-protons that are the background for Dark Matter searches.[3]. Furthermore, the low energy threshold of the p-p fusion reaction makes the $\mathrm{D} / \mathrm{p}$ ratio measurement useful to constrain propagation models at very low energies.

\section{The Instrument: AMS-02}

The Alpha Magnetic Spectrometer (AMS-02) [4-20] is a particle detector installed on International Space Station, whose components include a solid state tracker for determination of charge and rigidity, defined as $R=p / Z$ (where $p$ is the particle momentum), a Time of Flight detector (ToF) and a Cherenkov detector (RICH) for particle velocity measurement, a Transition Radiation Detector (TRD) and an Electromagnetic CALorimeter (ECAL) for hadron/lepton discrimination. The Tracker is composed by 7 layers inside the magnetic volume (inner Tracker), plus two external layers, one above the TRD and one above the ECAL (respectively, L1 and L9). A scheme of the AMS-02 detector is presented in Fig. 1, with all the main subdetectors highlighted.

Thanks to its wide acceptance and privileged position above the Earth's atmosphere, AMS-02 has been providing compelling science, having collected more than 170 billions of charged CR since 2011. Data from published AMS-02 results are online in the ASI/SSDC database [21]. 


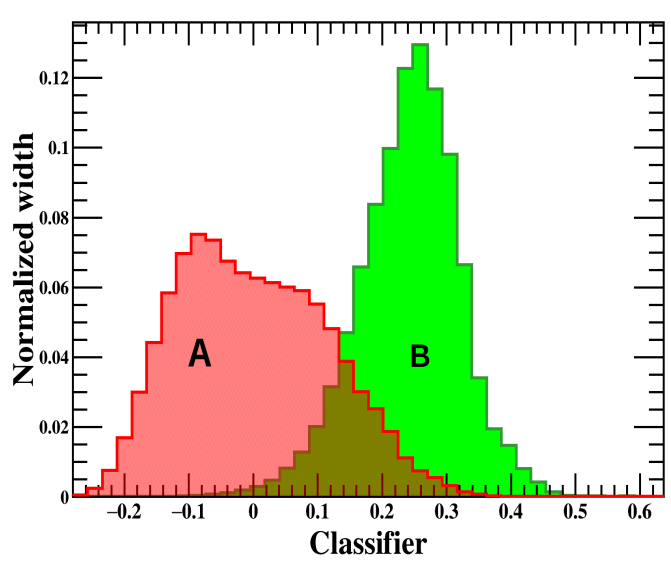

Figure 2: Distribution of RICH BDT classifier on background (A) and signal (B).

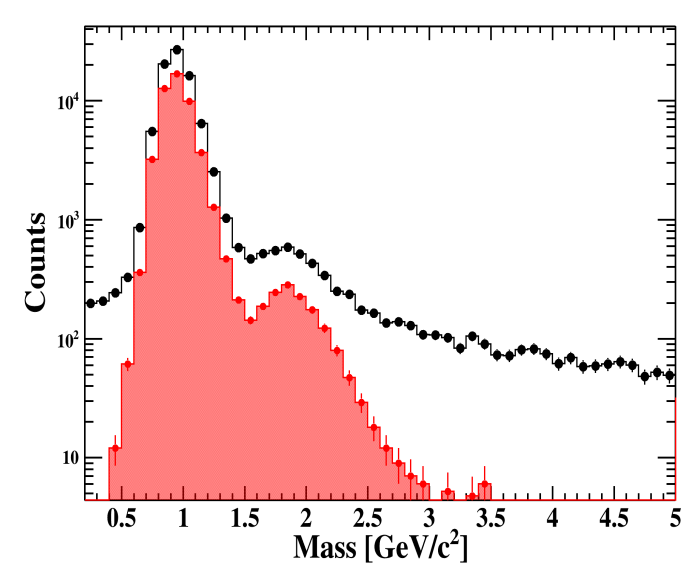

Figure 3: Mass distribution in a particular velocity bin of RICH velocity: before (black dots) and after (red distribution) RICH BDT cut.

\section{Isotope identification with AMS-02}

The identification of $\mathrm{D}$ from the proton component is performed with the concurrent measurement of Rigidity and velocity $(\beta=v / c)$, which gives a mass measurement through the relativistic formula:

$$
m=Z R / \gamma \beta
$$

This measurement is performed starting from a $Z=1$ sample, obtained with charge measurement at different levels within the detector. Additional selections about quality of rigidity and velocity reconstruction have been implemented to reject most of events suffering from interactions within the detector. In this analysis, the rigidity is measured by the inner Tracker, while the velocity by ToF and RICH. The $\beta$ resolution of ToF $(\sim 2-3 \%$ ) allows isotopic distinction up to $0.85 \mathrm{GeV} / \mathrm{n}$. At higher energies, AMS-02 RICH is used. This detector is equipped with two different radiators, Sodium Fluoride $(\mathrm{NaF})$ and Aerogel (Agl), with different values of thresholds and resolutions, allowing isotopic distinction in the Kinetic energy per nucleon range from 0.7 to $3.2 \mathrm{GeV} / \mathrm{n}(\mathrm{NaF})$ and from 2.7 to $8.9 \mathrm{GeV} / \mathrm{n}(\mathrm{Agl})$.

In the combined range $0.2-8.9 \mathrm{GeV} / \mathrm{n}$, the rigidity measurement has a rather constant resolution of $8 \%$, which dominates the overall mass resolution. Such limited resolutions prevent the eventby-event identification of isotopes and make a template fit approach, on the reconstructed mass distribution, necessary for both analyses. In general, the separation is performed dividing each range in narrow bins of measured $\beta$, to exploit the higher precision of the velocity measurement and minimize bin migration effects. The result is given in terms of rigidity on top of the instrument (T.o.I.) i.e. considering the particle energy loss that is not negligible in the low velocity region.

\subsection{Realistic Monte Carlo simulation}

The $\mathrm{p}$ and $\mathrm{D}$ templates have been obtained from a Monte Carlo (MC) simulation based on the GEANT4 package [22]. The detector resolutions in MC has been adjusted according to flight data 


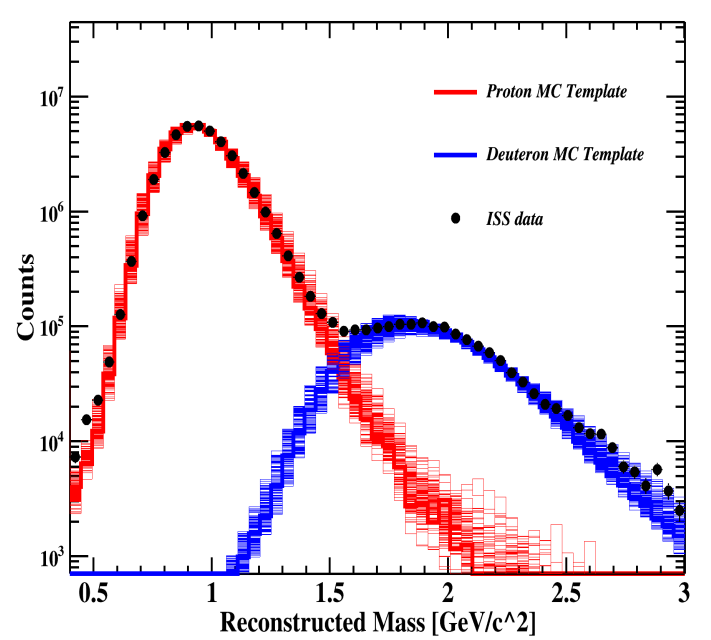

Figure 4: Collection of different templates for deuteron (blue) and proton (red) contributions in a typical mass distribution obtained with ToF detector.
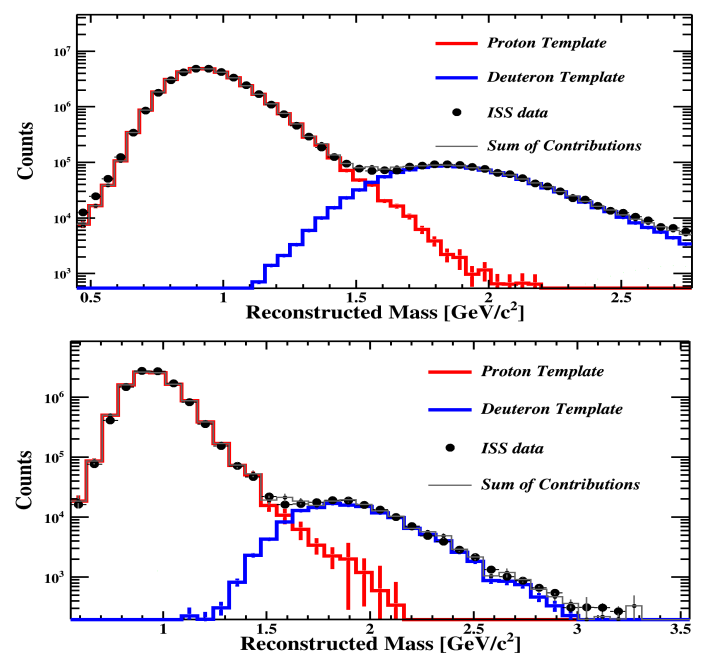

Figure 5: Example of final template fits of mass distributions in a particular bin of ToF (above) and RICH (below) energy region.

as explained in the following section, to provide matching with the data mass distributions. The measurement of the deuteron signal requires an accurate description of the proton mass distribution, which shows an high mass tail. Such tail is dominated by the migration of higher energy events towards lower energies, due to the saturation of the velocity value for relativistic particles. For this reason, a realistic spectral distribution in $\mathrm{MC}$ is fundamental to obtain template shapes compatible with the distributions from flight data. A further complication is the fact that the low energy spectrum of protons is time-dependent, due to the periodic solar modulation effect, so a timedependent re-weighting procedure of the MC spectrum was developed to account for this effect. As a result, the template shapes obtained from MC are able to describe both the proton peak and the proton tail in data at different times and modulation conditions.

\subsection{Mass distribution optimization}

The detector mass resolution has been improved by means of quality selections to reduce the amount of mis-reconstructed events. For the measurement in the ToF energy range, dedicated selections on the time of flight measurement, such as selections on the temporal and spatial consistency of the clusters, were optimized to reduce the background of mis-reconstructed particles. A geometrical matching was also required between the clusters in the ToF paddles and the track measured by the inner tracker.

In the RICH energy regions, a selection based on a multivariate BDT estimator was applied. In particular the number of photons produced by Cherenkov effect is proportional to $Z^{2}$, therefore only 3-4 photons are collected by RICH for charge-one particles and thus the quality of the measurement is easily affected by spurious hits, which turned out to be caused by back-splash electrons from ECAL. This effect is responsible for a high mass tail in the proton distribution overlapping the deuteron signal. A multivariate (BDT - Boosted Decision Trees) classifier was trained on a set of variables sensible to this effect, in particular the number of rejected hits in the RICH ring reconstruction and 
the ratio between the number of expected and measured photo-electrons for a charge-one particles. The training samples were selected directly from flight data, using the following criteria:

- Background: mass $>3.5$ (no stable $\mathrm{Z}=1$ particle is expected for $\mathrm{A}>2$ )

- Signal: $0.5<$ mass $<1.5$ (the bulk of proton signal)

A selection in the resulting classifier (Fig. 2) is able to increase the signal to noise ratio by more than one order of magnitude (Fig. 3).

\subsection{Deuteron identification}

The deuteron separation from proton background is performed fitting the data mass distributions. The templates for proton and deuteron components were obtained by MC simulation data-sets undergoing the same selections applied on data. Moreover, the resolution of each velocity-measuring sub-detector was fine-tuned to match the flight data.

To evaluate the systematic uncertainty in the result due to the template definition, a marginalization over the sub-detector resolution parameters was performed considering $(\sim 100)$ different configurations weighted by the parameter's likelihood probability (see Fig. 4). The figure 5 shows two examples of the fit performed respectively in the ToF and RICH energy regions.

\subsection{Evaluation of energy loss effect}

To obtain the D flux in cosmic rays, a correction for energy loss effects within the detector is necessary. The energy loss is caused by ionization within the detector before the inner tracker, mostly in TRD and ToF, and being $\propto 1 / \beta^{2}$, the fraction of this energy loss over the total is very different for different isotopes. The migration of proton and deuteron events towards lower velocity is thus very different, and prevents the use of a common measured beta regions to evaluate proton and deuteron flux as a function of $R_{T . o . I}$.

The change of velocity due to this energy loss was modeled using MC simulations (Fig. 6) with the parametrization:

$$
\Delta \beta=\frac{1}{m} \frac{\alpha}{\beta^{\gamma}}
$$

where $\alpha \simeq 0.0035$ and $\gamma \simeq 5.84$ are the parameters of the model. The effect of energy loss is accounted for each $R_{T \text {.o.I. }}$ and for a fixed particle, i.e. the $R_{T \text {.o.I }}$ interval is converted to $\beta_{T \text {.o.I }}$. using (1) and converted to a measured $\beta$ interval with (2). An independent mass fit is performed for every measured velocity bin for each isotope, using the methods described above.

\subsection{Evaluation of fragmentation effect}

The fragmentation reactions cause the production of secondary nuclei of $\mathrm{p}, \mathrm{D}$ and $\mathrm{T}$ from the fragmentation of primary ${ }^{3} \mathrm{He}$ or ${ }^{4} \mathrm{He}$, mostly due to interactions in the material of AMS-02.

In particular, the background coming from the fragmentation in the very small amount of material above layer 1 is not reducible and needs to be evaluated to avoid biases in the final flux. A pure data-driven methodology has been developed to evaluate such contribution. Exploiting the full statistics provided by AMS- 02 is possible to spot a relatively small contribution of $\mathrm{T}$ in 


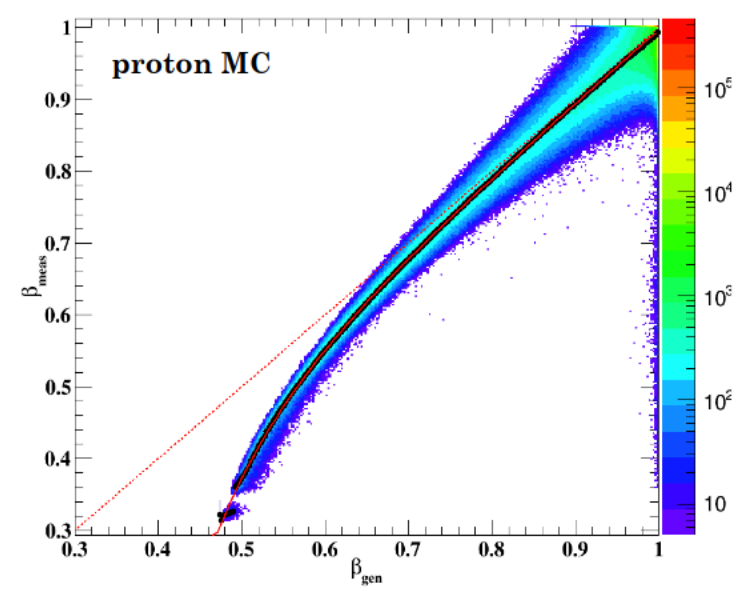

Figure 6: Effect of energy loss on reconstructed velocity of MC generated protons. The relation between measured $\beta$ vs $\beta_{\text {T .o.I. Was modeled in MC (red curve) }}$ and used for the energy loss correction as described in the text.

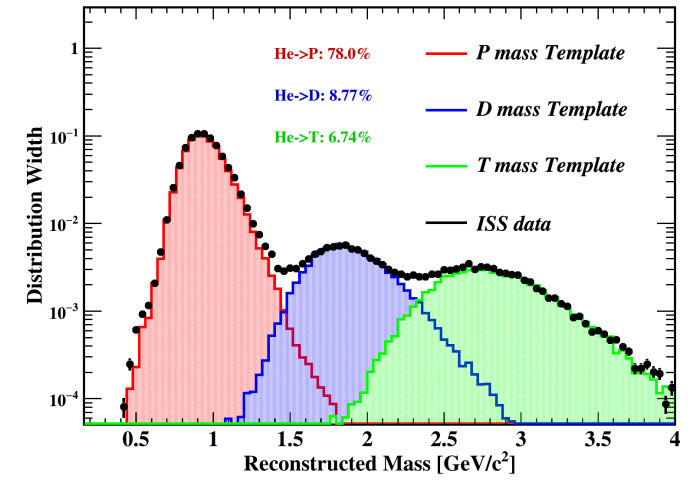

Figure 7: Example of the mass distribution of $\mathrm{He}$ fragments, obtained after the subtraction procedure described in the text. The distribution is fitted with three contributions: $\mathrm{p}, \mathrm{D}$ and $\mathrm{T}$ (Tritium).

the data mass distribution of cosmic charge-one particles. Since $\mathrm{T}$ is a relatively fast decaying isotope $\left(\mathrm{T}_{1 / 2}=12.3 \mathrm{y}\right)$, it is believed to be substantially absent in galactic cosmic rays, and thus it is produced by the above mentioned interactions. For this reason, its amount can be used as a measurement of the rate of $\mathrm{He}$ fragmentation processes. To measure the relative quantity of the other two isotopes, a measurement of the branching ratio of the He fragmentation reaction is necessary. To obtain this, we select a $Z=2$ sample with tracker L1, and we utilize TRD as target and Inner tracker as analyzer, fitting the mass distribution of the $Z=1$ events emerging from the the interactions in TRD. This allow to obtain a benchmark of the composition of the $Z=1$ contribution coming from Helium fragmentation (figure 7).
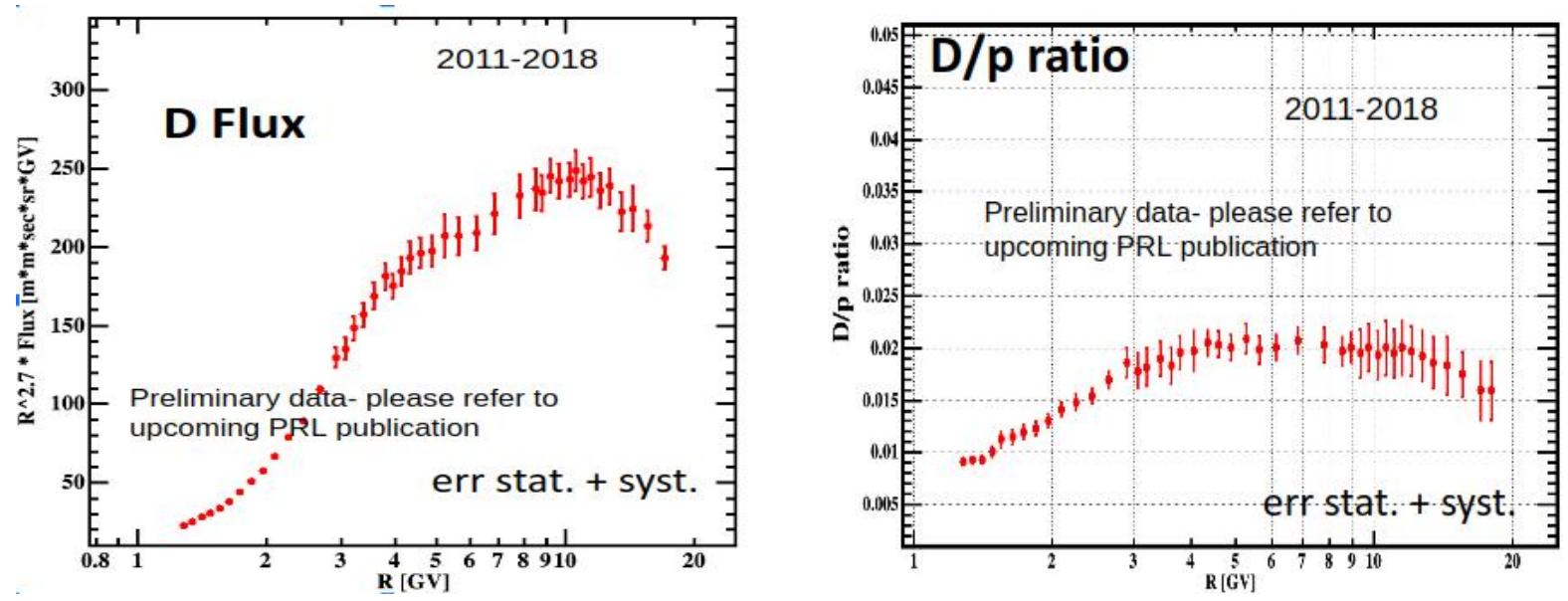

Figure 8: Preliminary measurement of the deuteron flux and the D/p ratio in CR. 


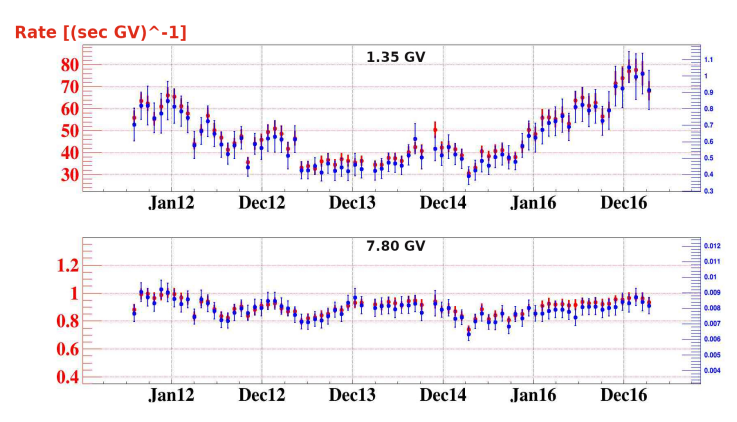

Figure 9: Preliminary: time dependence of the $D$ rate (blue) compared with the p one (red) in two different rigidity bins.

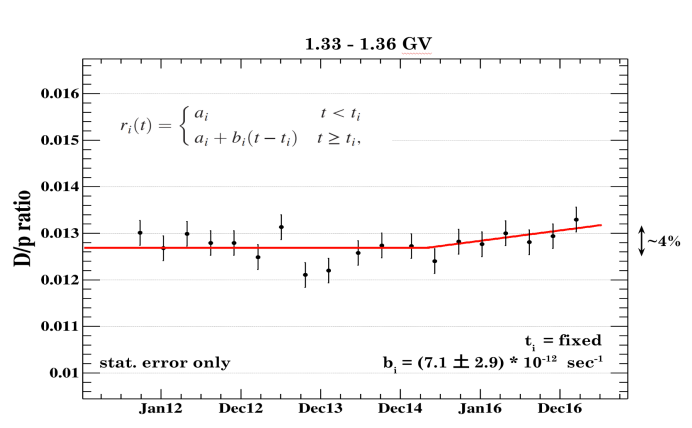

Figure 10: Preliminary: time dependence of the $D / p$ rate ratio in the $1.33-1.36 \mathrm{GV}$ rigidity range,In the fit, $t_{i}$ was fixed to the February 28, 2015 value [16].

\section{Conclusions}

The D flux in CR measured by the AMS-02 experiment extends in energy regions substantially uncharted by the previous measurements over a total period of 7 yrs all over the $24^{\text {th }}$ solar cicle, and present an accuracy of the order of few percent (Fig. 8). The measured D and p rates were normalized with the detector acceptance, calculated through MC simulations and validated with data-driven techniques. A systematic uncertainty was associated to this procedure, which dominates the total uncertainty on $\mathrm{D}$ and $\mathrm{p}$ fluxes. The high acceptance of the instrument allowed to measure the time dependence of the $\mathrm{p}$ and $\mathrm{D}$ fluxes at different rigidities (Fig. 9), using time bins corresponding to one single Bartel rotation ( $\sim 28$ days). In general, the overall cosmic ray flux is anti-correlated with solar activity. On top of this general behaviour, the D flux shows the same time structures of the proton one. In particular, first hints of a small time dependence of the $\mathrm{D} / \mathrm{p}$ flux ratio were found in the extremely low rigidity bins. Such time dependence is shown in Fig. 10 and modeled with the relation reported in the same figure. Its maximum amplitude in the period analyzed is $\sim 4 \%$.

\section{References}

[1] Coste B et al. 2012 Astronomy Astrophysics 539

[2] Tomassetti N, Feng J 2017 Astrophysical Journal Letters 835

[3] Gomez-Coral D et al. 2018 Phys.Rev. Lett D 98023012

[4] AMS Official website https://ams02.space/

[5] Aguilar M et al. 2013 Phys. Rev. Lett. 110141102

[6] Accardo M et al. 2014 Phys. Rev. Lett. 113121101

[7] Aguilar M et al. 2014 Phys. Rev. Lett. 113121102

[8] Aguilar M et al. 2014 Phys. Rev. Lett. 113221102

[9] Aguilar M et al. 2015 Phys. Rev. Lett. 114171103 
[10] Aguilar M et al. 2015 Phys. Rev. Lett. 115211101

[11] Aguilar M et al. 2016 Phys. Rev. Lett. 117091103

[12] Aguilar M et al. 2016 Phys. Rev. Lett. 117231102

[13] Aguilar M et al. 2017 Phys. Rev. Lett. 119251101

[14] Aguilar M et al. 2018 Phys. Rev. Lett. 120021101

[15] Aguilar M et al. 2018 Phys. Rev. Lett. 121051103

[16] Aguilar M et al. 2018 Phys. Rev. Lett. 121051101

[17] Aguilar M et al. 2018 Phys. Rev. Lett. 121051102

[18] Aguilar M et al. 2019 Phys. Rev. Lett. 122041102

[19] Aguilar M et al. 2019 Phys. Rev. Lett. 122101101

[20] Aguilar M et al. 2020 Phys. Rev. Lett. 124211102

[21] ASI/SSDC cosmic ray database https://tools.ssdc.asi.it/CosmicRays/

[22] Geant4 Website https://geant4.web.cern.ch/ 